\title{
Using conceptual blending to describe how students use mathematical integrals in physics
}

\author{
Dehui $\mathrm{Hu}^{1,2}$ and N. Sanjay Rebello ${ }^{1, *}$ \\ ${ }^{1}$ Department of Physics, Kansas State University, Manhattan, Kansas 66506, USA \\ ${ }^{2}$ School of Physics and Astronomy, Rochester Institute of Technology, Rochester, New York 14623, USA
}

(Received 29 March 2013; published 12 November 2013)

\begin{abstract}
Calculus is used across many physics topics from introductory to upper-division courses. The fundamental concepts of differentiation and integration are important tools for solving real-world problems involving nonuniformly distributed quantities. Research in physics education has reported students' lack of ability to transfer their calculus knowledge to physics. In order to better understand students' deficiencies, we collected data from group teaching or learning interviews as students solved physics problems requiring setting up integrals. We adapted the conceptual blending framework from cognitive science to make sense of the ways in which students combined their knowledge from calculus and physics to set up integrals. We found that many students were not able to blend their mathematics and physics knowledge in a productive way though they have the required mathematics knowledge. We discussed the productive and unproductive blends that students created when setting up integrals. The results of the study also suggested possible strategies to shifting students' constructing of blends to more powerful ones.
\end{abstract}

DOI: 10.1103/PhysRevSTPER.9.020118

PACS numbers: 01.40.- d

\section{INTRODUCTION}

Developing the ability to use mathematics is necessary to understand subject matter throughout science and engineering courses. Using mathematics in physics requires more than the straightforward application of algorithms and rules that students may learn in their mathematics classes. Previous work in physics education research has documented students' difficulties with mathematical fluency in physics problem solving [1-4]. In calculus-based physics courses, this issue is of particular concern as problems often require the application of sophisticated mathematical concepts such as differentiation and integration. To facilitate students' use of mathematical integration in physics, we need to first investigate how students solve physics integration problems and understand students' reasoning processes. The goal of our study is to adapt the framework of conceptual blending to systematically investigate how students set up mathematical integrals in physics and to get a clearer understanding of their difficulties.

Integration problems in physics involve blending of the physical world with mathematical symbols and concepts. There are often many different ways to interpret and apply mathematics in a given physical situation. Hence, there are various types of blends that students might construct in a given problem. In this paper, we use the cognitive framework of conceptual blending [5] to

\footnotetext{
*srebello@phys.ksu.edu
}

Published by the American Physical Society under the terms of the Creative Commons Attribution 3.0 License. Further distribution of this work must maintain attribution to the author(s) and the published article's title, journal citation, and DOI. describe the ways in which students set up mathematical integrals in physics contexts. The conceptual blending framework views the process of learning as selective projection and combination of knowledge from several different mental spaces. In our study, we conducted a detailed analysis of students solving a physics problem to investigate how they make connections among mathematical symbols and concepts, as well as concepts associated with the physical world. We make comparisons of the blends created by students and discuss the effectiveness of certain kinds of blends in facilitating students' use of integration in a productive way. Identifying the different types of blends that students create provides a way of parsing students' reasoning when approaching physics integration problems.

Previous studies often investigated students' difficulties with particular facets of using integration, such as recognizing when to use integration $[3,6]$, finding the infinitesimal expression for a physical quantity [4,7], and deciding the limits of an integral [3,4]. Our study describes the overall strategies students used to apply integrals in physics contexts through the perspective of mental space integration. This framework allows us to organize the bits of knowledge that exist in student reasoning and connect the fine-grain-sized knowledge elements to the entire picture of student approach.

In the next section, we provide an overview of previous work on theoretical development of mathematical use in physics. In Sec. III, we describe the conceptual blending theoretical framework and how it is used in our study. In Sec. IV, we focus on the methodology used to collect and analyze our data, and in Sec. V, we present the results of our study. Finally, in Sec. VI, we discuss our results and implications for teaching and education research. 


\section{BRIEF OVERVIEW: STUDENTS' USE OF MATHEMATICS IN PHYSICS}

To characterize and understand how students use mathematics in physics, researchers have utilized several frameworks to analyze student behavior. In this section, we briefly review some of the recent work in this area.

Sherin [8] introduced symbolic forms (i.e., cognitive mathematical primitives) to understand student interpretation of mathematical equations in physics problem solving. Symbolic forms allow students to associate meanings with certain structures of mathematical expressions. A symbolic form has two components: a symbolic template (e.g., []$=[],[] /[]$ ) and a conceptual schema. The conceptual schema is "a simple structure associated with the symbolic form that offers a conceptualization of the knowledge contained in the mathematical expression" [8]. Sherin observed that students not only applied known equations or given principles, but also invented their own equations from intuition. When observing five pairs of intermediate-level engineering students solve physics problems, Sherin discovered the existence of symbolic forms based on the fact that students "learn to associate meanings with certain structures in equations." One problem was based on the physical scenario that a person gives a block a shove so that the block slides across a table and then comes to rest. Students were asked to talk about the forces acting on the block and discuss what would happen if the block was heavier. A pair of students, Mike and Karl, invented an equation $\mu=\mu_{1}+C\left(\mu_{2} / m\right)$ for the coefficient of friction based on their understanding of the situation "the coefficient of friction has two components: one that's a constant and one that varies inversely as the weight." This expression was not from the textbook; instead, they constructed this equation from their understanding of the physical scenario. One symbolic form identified by Sherin from this expression was "parts-ofa-whole." The symbol template that two or more terms are separated by plus $(+)$ signs contains a conceptual schema "a whole is composed of two or more parts." Sherin's symbolic forms were primarily based on student reasoning of algebraic equations.

Meredith and Marrongelle [6] later applied symbolic forms to analyze student understanding of equations in calculus-based physics problems. They identified several symbolic forms that cued students to use integration, including the dependence cue and the parts-of-a-whole cue. The "dependence" symbolic form is associated with the mathematical entity of function $[\ldots x \ldots]$. The associated structure of the parts-of-a-whole form is the Riemann sum (i.e., []$=[]+[]+\cdots$ ). They categorized the symbolic forms that appeared at the beginning of the problem solving process when students decided whether or not to use integration.

Jones [9] extended the symbolic forms framework to analyze student understanding of the integral concept.
He interviewed nine students who were enrolled in an introductory-level physics course which was designed primarily for students in physics and engineering. All students were interviewed twice in their study. In the first interview, students were given open-ended mathematics problems related to integrals; in the second interview, students were given open-ended physics integration problems involving real-world objects. He identified four major symbolic forms associated with the integral symbol template " $\int_{[]}^{[]}[] d[]$." The area symbolic form basically takes the integral expression and interprets it as an area in the $x-y$ plane. The adding up pieces symbolic form refers to the evidence that students sliced the area on a graph into infinitely small pieces and added those pieces to find the total amount. However, Jones argued that under this symbolic form, the limiting process occurred before the addition process took place. Thus, this symbolic form diverged from the Riemann sum process. The function mapping symbolic form conceives the integral as a "pairing of objects," which matches the integrand with an "original function." According to the problematic add up then multiply symbolic form, the first box inside the integral $\int_{[]}^{[]}[] d[]$ was added up over the infinitesimally small pieces and the resultant summation was then multiplied by the quantity represented by the differential. Among the four major symbolic forms, Jones concluded that the adding up pieces symbolic form is a more productive way to view the integral in both mathematics and physics contexts.

Tuminaro and Redish [10] used the framework of epistemic games to describe students' use of mathematics in physics. Collins and Ferguson [11] introduced epistemic games to describe expert scientists' approaches to scientific inquiry. Epistemic games have two structural components: the entry or ending conditions and moves. The entry and ending conditions determine when it is appropriate to play that game. When solving physics problems, students' perceptions about physics problems determine the entry and ending conditions. The moves are the steps that can be taken in the game. Tuminaro and Redish [10] identified six different epistemic games that students played while using mathematics in algebra-based physics problems: Mapping Meaning to Mathematics, Mapping Mathematics to Meaning, Physical Mechanism game, Pictorial Analysis, Recursive Plug-and-Chug, and Transliteration to Mathematics. In the Mapping Meaning to Mathematics game, for example, they identified five basic moves: develop a story about the physical situation, translate quantities in the physical story to mathematical entities, relate the mathematical entities in accordance with the physical story, manipulate symbols, and evaluate the solution. A student's decision to play a particular game is determined by preconceived expectations about problem solving in physics.

Extending Tuminaro and Redish [10], Gire et al. [12] used the framework of epistemic games to characterize five 
students' use of graphs to solve physics problems and the hints given by an instructor to facilitate the students' problem solving. They introduced the Graphical Analysis game and identified the moves students made. They found that students demonstrated facility with the interpret lexical information, readout a value, and calculate area moves within this epistemic game. The create a story move was the most difficult move for these students, in terms of both connecting the graph to the story situation and connecting the graph to concepts leading to a situation.

These previous studies using symbolic forms and epistemic games frameworks have provided ways for educators and education researchers to describe students' ideas as students use mathematics in the context of physics. Symbolic forms provide us with a better understanding of students' conceptual schema as they deal with mathematical equations and other symbolic expressions. Based on the knowledge-in-pieces tradition, the symbolic forms are kinds of mathematical resources that students bring from their prior learning experience. The epistemic games from Tuminaro and Redish [10] and Gire et al. [12] depict the common reasoning patterns in student use of mathematics in physics problem solving. The purpose of epistemic games is not to describe how students solve certain physics problems but to help us understand how students perceive the use of mathematics in physics. For example, in the Mapping Meaning to Mathematics game, an important feature of this game is that students relate a physical situation to mathematical entities and make sense of mathematics in the physics scenario. However, it does not describe how a student interprets particular mathematics or physics concepts. The symbolic forms framework describes the conceptual schema associated with a certain mathematical template and it is based on the implicit assumption that resources are created and then applied in a situation. In our work, we are interested in how students apply their mathematics knowledge to a physics situation to set up integrals. In the following section, we describe our theoretical framework and demonstrate that it can serve a powerful paradigm to analyze student creation of knowledge from the integration of existing knowledge in distinct domains.

\section{CONCEPTUAL BLENDING FRAMEWORK}

We use the framework of conceptual blending, also called mental space integration by Fauconnier and Turner [13], to describe students' use of mathematics in physics. Conceptual blending or integration describes how the mind combines two or more mental spaces to make sense of linguistic inputs. Mental spaces are small conceptual packets or knowledge elements that tend to be activated together [14]. According to this framework, one creates new meanings from the combination of different mental spaces that share content or structure. Blending, as a general cognitive process, brings two or more spaces together through selective projection, taking some information from each input to compose a blend. The new space is called the blended space. The blend often inherits partial structure from input spaces but also has its own emergent structure [15]. The way a person blends several input mental spaces together depends strongly on the cues and contexts. Constructing a blend involves three operations: composition, completion, and elaboration.

Consider the phrase "computer virus," which we use frequently in our vocabulary nowadays. To understand this phrase, one has to blend knowledge from two different mental spaces-a biological mental space and a computer mental space. A biological mental space contains elements like a biological system, cell, virus, and immunity mechanism. A computer mental space includes elements like memory, software, and programs. In the blended space, a computer program and virus are fused into a new, single entity "computer virus" through the process of "composition," meaning that the computer virus is composed of elements from two input spaces. The composed structure often provides relations that are not available in the input spaces. When completing the blend, crucial elements from each input (e.g., viruses attack normal body cells, software gives instructions to a computer) are mapped to the third space to form the blend. Meanwhile, an organizing frame (i.e., the property of viruses-reproduction and attacking the normal system) is adopted to organize the knowledge elements in the blended space [16]. When the blend is complete, it can be elaborated to make inferences according to the rules in the blend. The process of elaboration is also called "running the blend." By elaborating on the knowledge in the computer virus blended space, a new meaning emerges - a computer virus as a computer program that can replicate itself and spread from one computer to another, usually making unauthorized and undesirable changes to the computer. Only in the blended space does the phrase computer virus make sense. In general, composition, completion, and elaboration lead to emergent structure in the blend; the blend contains a structure that is not preexisting in the input spaces [17].

The conceptual blending framework has also been extended to analyze students' mathematical and scientific reasoning. Zandieh et al. [18] applied the theory of conceptual blending to illustrate how university students construct mathematical proofs. Bing and Redish [14] used the conceptual blending theory to model how students combine physical and mathematical knowledge to construct solutions to physics problems. They described two representative ways in which students blend their knowledge from physics and mathematics mental spaces. A singlescope blend only involves unidirectional mapping of the elements from one input space into the organizing frame of the other while a double-scope blend involves an integration of the organizing frames from input spaces. In the context of using mathematics in physics problem solving, 


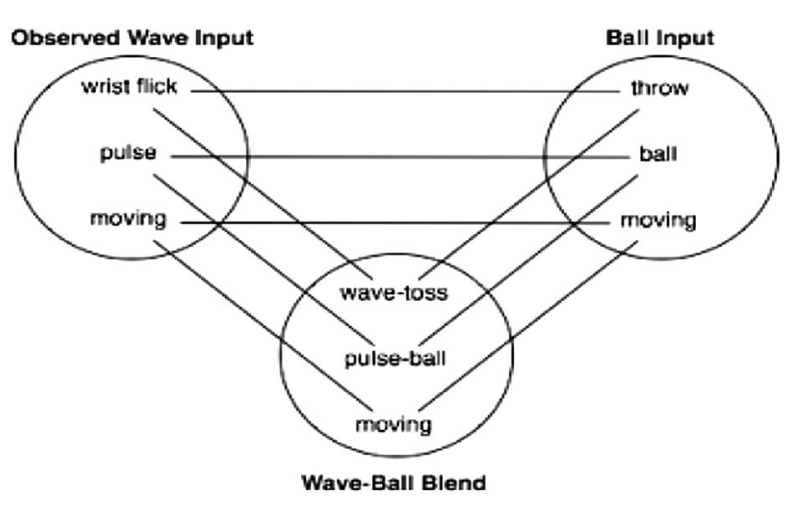

FIG. 1. Wave-ball blend from Wittmann [17].

a single-scope blend often refers to a one-way mapping of physical quantities to an existing mathematical equation or template. In a double-scope blend, students not only map an existing mathematical equation to a physics context, but also translate a physical scenario to a mathematical expression. They pointed out that the difficulties students experienced were often not from their lack of prerequisite knowledge but from inappropriate blending of mental spaces.

More recently, Wittmann [17] used the conceptual blending framework to describe emergent meanings in students' understanding of wave propagation. He analyzed gestural, perceptual, and verbal information to describe how different elements were combined to create new, emergent meaning. The "wave-ball" blend (Fig. 1) is formed by selective projection from two different mental spaces: observed wave pulse and imagined ball thrown in the air. Then he compared this blend with a "beadedstring" blend (Fig. 2) containing the input spaces of the observed spring and falling dominoes. The blend that students make is determined by their selective attention to parts of the observed physical system as they determine which part of the system to use in the process of projection from the input spaces to the blended space.

According to the cognitive blending theory of Fauconnier and Turner, learners must find a way to bridge or blend their knowledge in different mental spaces to

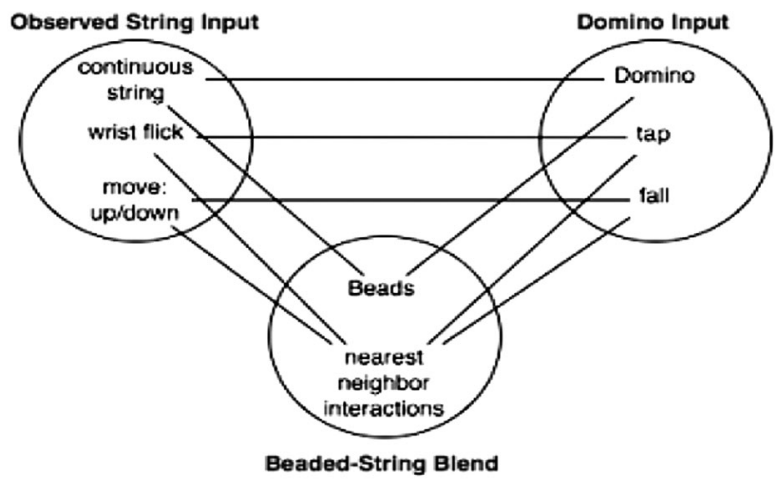

FIG. 2. Beaded-string blend from Wittmann [17]. make sense of new information. Using mathematics in physics requires the blending of mathematical ideas with physical situations. Experts often automatically and naturally blend their knowledge in mathematics and physics in efficient ways that they have developed through years of learning and experience. However, novice students might blend their knowledge in mathematics and physics in unproductive ways that hamper their physics problem solving ability. In this study, we use the tool of conceptual blending to analyze how novice students bridge their knowledge in two distinct worlds-calculus and physics. This analysis will provide us deeper insights into (a) what information students perceive as relevant, i.e., which knowledge elements of a problem do students attend to and which they ignore, when constructing a solution, and (b) how students interpret their solution, i.e., what new meanings they create when they combine their understandings of mathematics and physics. The former involves the process of selective projection of information from the mathematical and physical mental spaces, when composing a blend, and the latter involves the creation of an emergent structure when running a blend. In the computer virus example, when creating this blend, certain elements from each space - the computer space and the biological space-are selected to be projected into the blended space. When running the blend, a new meaning emerges: computer virus is an entity that can replicate and spread from one computer to another.

In the last section we reviewed two other frameworkssymbolic form and epistemic game, which also have explanatory power in understanding student use of mathematics in physics. A discussion about the three theoretical constructs might be helpful for understanding why we choose cognitive blending as our theoretical framework in this study. Symbolic forms are mathematical resources associated with mathematical equations. The knowledge elements in the input mental spaces can be described as resources. Thus, the formation of symbolic form can be described using the language of conceptual blending. However, after years of learning and practice, learners may often activate some symbolic forms as a unit (or a blend), for which the input mental spaces are no longer distinguishable and the blending process cannot be easily retraced. In this study we use the conceptual blending framework to describe how students organize their existing knowledge resources in a situation that they have little or no experience with.

Epistemic games describe students' cognitive reasoning patterns, and their purpose is to model the process component of physics problem solving, i.e., the moves that students make as they solve problems [19]. Resources are the knowledge base of epistemic games and are considered the ontological component for describing students' physics problem solving. The cognitive blending framework focuses on the conceptual component, describing the 
ways that students combine their knowledge in different mental spaces as well as the generation of new ideas that emerge from the combination. There appears to be a connection between the epistemic games that students play and the type of blends that they construct. For example, Bing and Redish [14] described the "double-scope" blend when students solve the air resistance problem. From the perspective of epistemic game, students might have played the Mapping Meaning to Mathematics or the Mapping Mathematics to Meaning game. However, one cannot predict what specific blends students create based on the epistemic games that they play. For example, we can imagine a student who plays the Mapping Meaning to Mathematics game is more likely to create a double-scope blend, but there are a number of ways for constructing a double-scope blend.

In general, the three theoretical constructs serve different purposes: the resources framework describes the ontological components, the epistemic game framework models the process component, and the conceptual blending framework models the creation of meaning that is emergent as students' use mathematics in the context of physics problem solving.

\section{METHODOLOGY}

\section{A. Data collection}

Data for this study were collected as part of a semester long series of teaching or learning interviews [20] during an introductory-level calculus-based physics course for engineering majors at a U.S. Midwestern university. This course is the second semester of a two-semester sequence. The first semester mainly focused on mechanics topics and the second semester mainly focused on electricity and magnetism (E\&M) topics. This course has two main components-two 50-minute traditional lectures with clicker questions and two 110-minute integrated laboratory and problem solving sessions (i.e., studio sessions) [21]. The class has an enrollment of about 250 students who are divided into several studio sessions with a maximum of 40 students each.

Thirteen students were selected from a pool of 40 volunteers, depending upon scheduling convenience.
The selected participants were organized in small groups of three or four students each, to discuss physics problems on a whiteboard. Each group met with the interviewer separately over eight 75-minute long sessions that occurred approximately biweekly throughout the semester. All participants had taken prerequisite calculus classes before the physics course. Interview problems presented to students were $\mathrm{E} \& \mathrm{M}$ problems requiring integrals. During the small group discussion, students discussed problems in groups on a whiteboard and the interviewer watched students work. The interviewer was a silent observer for a vast majority of the time, and only interjected to ask students to explain their thinking whenever necessary. When the students were unable to proceed, the interviewer would engage in Socratic dialog in order to provide hints and cues to facilitate the participants in figuring out the next step in the solution process. The interviewer refrained from providing any instruction or feedback during the session. Our data sources include the video-recorded conversations of students and their work completed on the whiteboard.

\section{B. Data analysis}

In this study we present a detailed analysis that focuses on one physics problem in the context of resistance. The problem (Fig. 3) was presented to students during the fifth interview session. There were two main reasons for choosing this problem for a detailed analysis. First, students were familiar with the resistance concept, but they had not seen any specific example nor had they received any instruction on how to find the resistance of a resistor with nonuniform resistivity. Students had to invent their own integral equation as there was no specific integral equation on resistance that they could draw upon from their memory. Second, students used diverse strategies and reasoning when solving this problem. This problem seemed neither too easy nor too difficult for most students. Most students were able to invent strategies to solve this problem and explain their thinking.

The problem (Fig. 3) involves finding the total resistance due to a cylindrical resistor with nonconstant resistivity. When the resistivity is not a constant, one must first chop

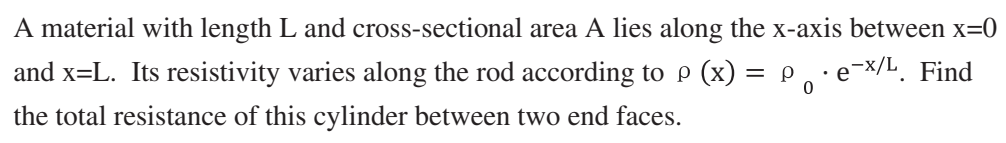

FIG. 3. Interview task. 


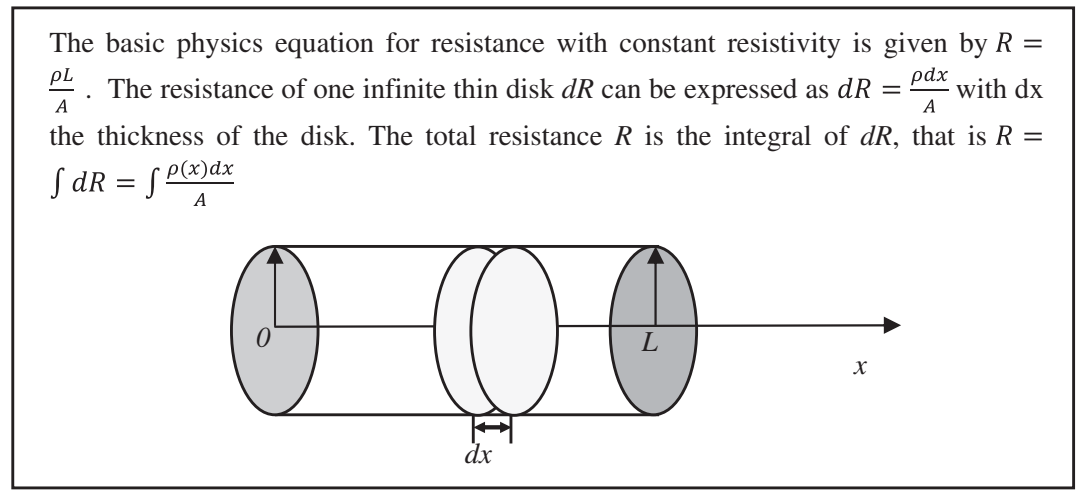

FIG. 4. Partial solution to the interview task in Fig. 3.

the cylinder into infinitesimally thin disks and find the resistance $d R$ of each disk (Fig. 4). We find the total resistance by summing up, i.e., integrating over the resistance $d R$, of each disk. Finally, we substitute the resistivity function $\rho(x)$ provided in this equation and evaluate the integral.

Our unit of analysis was a student discussion group. We transcribed the video files for all five groups working on the task. First, we conducted a qualitative analysis using a phenomenographic approach [22]. An important aspect of our approach was that we did not categorize students' descriptions based on predecided categories. Rather, the categories emerged from the data. Then, we identified the important themes that emerged from students' conversations or written work on the board. Next, we examined these emergent themes through the lens of the conceptual blending framework. For each theme, we determined the input spaces and blended spaces by analyzing the ways in which students connected their ideas from different domains. Finally, we generated a holistic description of how the students blended their ideas. This holistic picture describes how the knowledge elements from each input space are projected to the blended space and how a new structure emerges from the blend.

\section{RESULTS AND DISCUSSION}

In this section, we present four distinct conceptual blends identified from five groups of students working on the problem (Fig. 3). We noticed that students within the same group did not necessarily follow the same approach; thus, there was often more than one blend created in a group. We claim that the blending process is a dynamic process. That is, students do not have explicit ideas in their mind and construct the solutions spontaneously. We found that students constructed one blend at the beginning and later changed the blend as they proceeded. Some blends eventually led students to solve the problem correctly while others hampered students' efforts in setting up the correct integral. We will illustrate the four different blends identified from student work.

\section{A. Blend A}

Two groups of students constructed the same blend. We discuss one group of three students as an example. Prior to this dialog, all three students set up their own equations and they all seemed to be in agreement with each other. We show the solution from Phil in Fig. 5. The interviewer then prompted the students to explain their thinking, which resulted in two of the students (Zad and Alan) participating in the conversation below.

Interviewer: Can you guys sort of explain what your plan is for solving this problem?

Alan: Well, we set up our basic equation. And then we are using our $\rho(x)$ that they gave us. We are going to integrate that to give the whole area, right? That's where we are at right now.

Interviewer: What do you mean by "integrate to get the whole area"? Can you talk more about that?

Alan: We are looking for total resis (pause) $\rho$ total, yeah, and this (points to the resistivity function in the problem statement) gives us $\rho$ at any one point, so we are summing up all of its points.

Then students discussed the cross-sectional area $\mathrm{A}$ in the integral and calculated the integral. After they were all done, the interviewer continued:

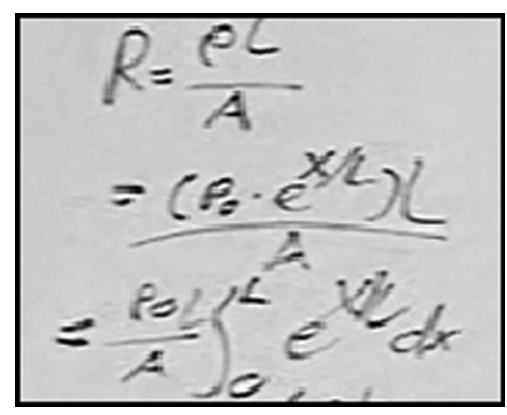

FIG. 5. Equation set up by Phil. 
Interviewer: So can you guys explain your solution from the beginning?

Zad: $\quad$ Resistivity times length over area. We have function of resistivity, pull out all the constants. Length is a constant, $x=L, \rho_{0}$ is a constant, over $A$, we need to take the integral of $x$, which, a function of $x$, which is $e^{-x / L}$, from 0 to $L$, because you are taking from 0 to the length L... (Continues to talk about his calculation.)

Interviewer: Can you guys explain what the meaning is for the integral part? Is there a meaning for that?

Zad: $\quad$ Um, it gets more resistive as it approaches $L$. That's a function of $\rho$ but just the resistance isn't constant, I guess? So you have to take, since it's not constant, you have to take the total integral of ... sum all the way across.

When working on this problem, this group of students mainly focused on mathematical manipulations and looked for ways to relate mathematical symbols to a mathematical equation. First, the students related the resistivity function $\rho(x)$ to the basic resistance equation $R=\rho L / A$ by substitution. In mathematics, substitution or "plugging in" is one of the most basic operations, often made subconsciously by students even though the resulting equation does not have any concrete meaning in this physical situation. Students also demonstrated a basic knowledge of a function. Alan explained that the resistivity function "gives us $\rho$ at any one point" and Zad talked about "it gets more resistive as it approaches L." In their explanation, Alan talked about "summing up all of its points" and Zad said "take the total integral of, sum all the way across." Hence, students had the notion that "an integral represents a sum."

When setting up integrals, students' reasoning involved three input spaces: symbolic space, mathematical notion space, and the physics space. A blending diagram is shown in Fig. 6. The symbolic space includes abstract mathematical symbols and notations, such as resistivity function $\rho(x)$ and resistance equation $R=\rho L / A$. The mathematical notion space involves students' knowledge about mathematical concepts and notations. In this case, the mathematical notion space includes students' ideas of the function as being nonconstant, integral $\int$ as a sum, and $d[]$ as the variable of integration. The physics space includes physical quantities associated with the object, such as resistivity, resistance, and length. It is not our purpose to make a comprehensive list of the elements in each space, rather, only the most relevant elements are included for the purpose of describing students' reasoning. For example, the area of the cylinder is also a feature of the physical object in the physics space, but it is not included as it seems less relevant in students' reasoning process.

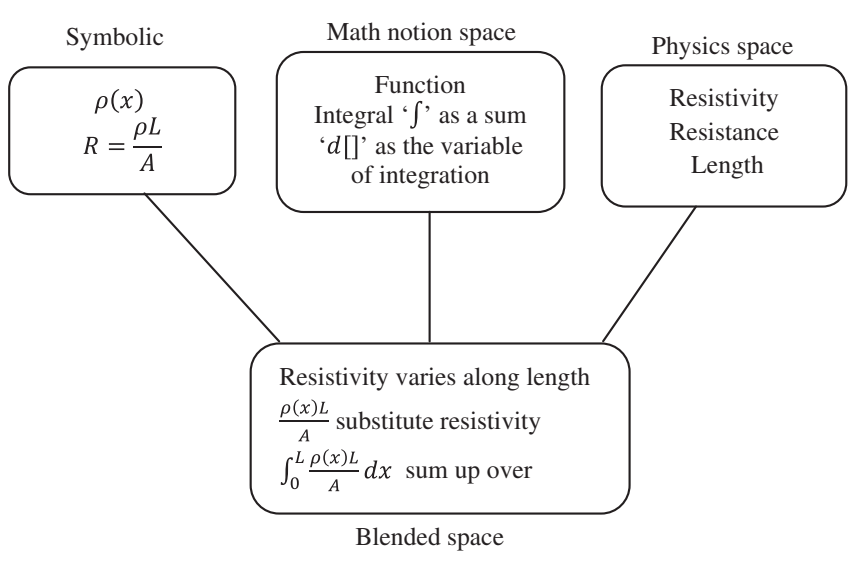

FIG. 6. Blend A.

Elements from input spaces were projected into a new space-the blended space. In the blended space, the expression $\rho(x)$, the notion of function as a nonconstant quantity, and the cylindrical resistor were combined, generating a meaning that the resistivity varies along the axis of cylinder. Students seemed to map the resistivity function $\rho(x)$ and the basic resistance equation $(R=\rho L / A)$ into the new space to create a new expression $[R=$ $\rho(x) L / A]$. Then the notions of an integral $\int$ as a sum (i.e., performing an integral would produce the total quantity) and differential $d[]$ as variable of integration were projected into the blended space. Guided by this notion, students integrated the expression of resistance that they obtained earlier over the variable $x$. A new meaning seems to emerge from this blend-the total resistance is the sum of a varying resistance across the length over which the resistivity varies. This new meaning is generated from the blend and it did not preexist in any of the input spaces. Students started out with basic information (i.e., physical world and mathematical symbols) given in this problem and blended the information with their notion of relevant mathematical concepts. The organizing structure was based on the notion of an integral in mathematical notion space as representing a sum. The elements in the physics space were imported into the organizing structure without considering whether or not the way the elements were structured was appropriate. For example, when integrating the new expression $R=$ $\rho(x) L / A$, the resistance element was mapped into the blended space, giving a meaning of summing up resistance. However, students neither talked about which resistance (i.e., the resistance of which part of the object) this $R$ represented nor talked about what it meant to "sum up the resistance" physically. In other words, it is a one-way mapping, importing elements from the physics space and symbolic space into the structure of math notion space. Hence, students did not recognize that the expression $R=\rho(x) L / A$ was incorrect in this physical situation. All students said they were confident about their answer. 


\section{B. Blend B}

When solving this problem, this group of students first constructed a blend as discussed in the last episode. At the very beginning of their problem solving, one student ( $\mathrm{Zad})$ wrote " $d R$ " but he did not specify what $d R$ meant nor had he found the expression for $d R$. In the following episode, the interviewer asked students to explain what $d R$ means and they started to find the expression for $d R$. Students eventually constructed another blend which was different from the blend they had originally created. When prompted to explain the differential term $d R$, students' notion about the differential template $d[$ ] was activated. Hence, students shifted from one blend to another.

Interviewer: So here I saw you wrote $d R$. Can you sort of explain what do you mean by this $d R$ ?

Zad: $\quad$ Like electric field, anything else uneven, we have to define the integral of $d R$. We have to find $d R$, um ... with ... over an area.

Alan: $\quad$ It's a small R. It's just a small part of our resistance (points to the cylinder of this problem). Like, we just look at a section of it. We look at ... just on our cylinder like we are looking at our resistance at this point right here (points to the dot on the central axis of the cylinder in Fig. 7). It would be just our dR there. So we do that and then we take the integral ... adds up the little parts together.

Interviewer: How would you set up the equation for $d R$ ?

Then students started to set up the equation for $d R$ and talk about their thinking at the same time.

Alan: $\quad$ So $d R$ would be equal to ... (Fig. 8), but I need $d x$, I guess.

Zad: $\quad$ We would have to take the derivative of this one (points to the equation in Fig. 9). Um,

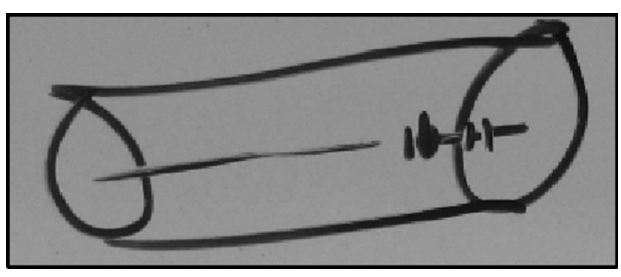

FIG. 7. Picture drawn by Alan.

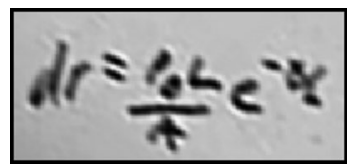

FIG. 8. Equation set up by Alan.

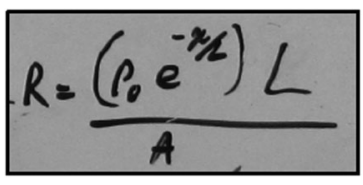

FIG. 9. Equation set up by Zad.

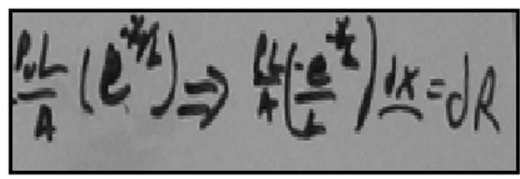

FIG. 10. Expression for $d R$.

this is the derivative. Take the derivative of that (Fig. 9). Would that (Fig. 10) be the derivative of it?

Alan: $\quad$ It should equal $d R$.

Zad: $\quad d R \ldots$ cause that will times $d x$, not equals $d R$. So, instead, you have to take the derivative of something, we just plug in to $d R$ (sets up equation in Fig. 11).

Interviewer: So why did you take the derivative of this one (points to Fig. 9)?

Zad: $\quad$ Um, because we just plugged it in to ... we plugged what $R$ was into the integral of $R$ basically. So, we need to take the derivative of it. So, we can plug in to R because we basically pull dx out of nowhere, because the derivative of the only changing function ... then we require $d x$. We need to integrate that.

At the beginning of this conversation, when asked to explain what $d R$ meant, Zad said, "like electric field ..., you have to define the integral of $d R$." We noticed that Zad did not actually talk about the meaning of $d R$. Instead, he recalled the electric field problems in which the total electric field $\vec{E}$ is the integral of $d \vec{E}$. Then he argued that the total resistance $R$ should be the integral of $d R$. Hence, his argument seemed to be based on pattern matching with similar examples he had seen before rather than understanding what $d R$ was and how it was related to the total resistance $R$. Alan seemed to have a more concrete understanding that $d R$ represented " a small $R$ " or " a small part of our resistance." He drew a picture (Fig. 7) to explain "resistance at this point." When finding the expression for $d R$, Alan just plugged the resistivity function into the basic

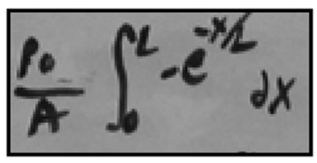

FIG. 11. Expression for total resistance. 
resistance equation (Fig. 8) and then he realized there must be something wrong with this equation because he "need [ed] $d x$." Zad proposed a different approach. He first substituted the resistivity function into the basic resistance equation to get a new expression for " $R$ " (Fig. 9), then he took the derivative of this new expression with respect to $x$ to get the differential of $R$, which was $d R$ (Fig. 10). Finally, they set up a definite integral of $d R$ to find the total resistance (Fig. 11). When finding the expression for $d R$, Zad explained "that will times $d x$... you have to take the derivative of something." We suspect that Zad realized $d x$ was needed in the differential equation (Fig. 8) set up by Alan and this prompted him to "take the derivative of something" in order to get $d x$. In this problem, Zad activated the mathematical operation of "taking the derivative" to find the differential expression $d R$. Upon seeing Zad's approach, Alan erased his work and seemed to accept this approach.

We noticed that Alan and Zad explained $d R$ in different ways. However, Alan did not propose another approach for solving this problem based on his own understanding. Hence, the blending diagram (Fig. 12) is primarily based on Zad's work. Three mental spaces were involved: symbolic space, mathematical notion space, and physics space. The symbolic space includes the expression of resistivity function $\rho(x)$, the basic resistance equation $R=\rho L / A$. The math notion space includes the mathematical concept of function, differential $d$ as the operation of taking the derivative or differentiation, and the integration-differentiation relation (i.e., integration is the reverse operation of differentiation) contained in the form of $\int d[]$. The physics space includes physical quantities of resistivity and resistance.

The expression of $\rho(x)$, the notion of function as a nonconstant quantity, and the physical quantity of resistivity were projected into the blended space, and the blend conveyed the information of uneven resistivity. This uneven property further cued $\mathrm{Zad}$ to use integration. The basic resistance equation was mapped into the mathematical structure of $d[]$ and $\int d[]$ in the blended space. Under

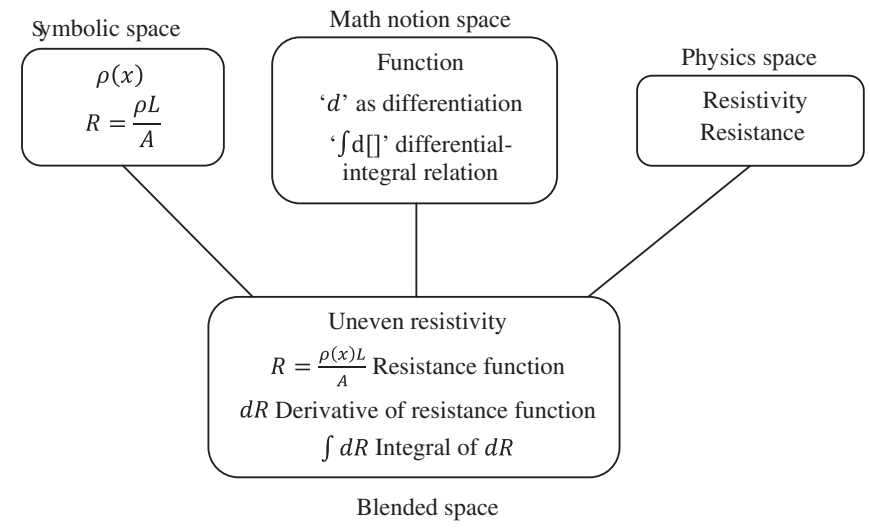

FIG. 12. Blend B. the notion of differentiation and integration operations, students created a new expression for resistance so that they could take the derivative of the resistance function and then take the integral of the differential resistance. The new resistance function was obtained from a direct "plug-andchug"- plugging in the resistivity function into the basic resistance equation. In the blended space, a new structure emerges: the total resistance is the integral of the differential resistance, which is the derivative of a resistance function. The organizing structure of this blend was recruited primarily from the mathematical notion space. Similar to blend A, this blend was constructed under a oneway mapping, in which the elements in physics space and symbolic space were mapped onto the mathematical structures of differential $d[]$ and integral $\int d[]$. Students did not map the resistance function, the differential resistance, and the integral to the physics space. Even though the interviewer prompted students to explain their thinking several times, Zad still did not map the solution back into the physics space and eventually Zad responded "I do not know how to explain it other than mathematically." The solution appeared reasonable for him; however, he was unable to explain the physics underlying the solution or recognize that it was incorrect.

\section{Blend C}

In the episode below, two students (Jared and Lee) used different approaches to solve this problem. We will discuss Jared's approach in this example. Jared started with the basic resistance equation and then converted it into an integral form as shown in Fig. 13. When he completed this equation, the interviewer prompted him to explain his thinking. Below is a transcript from the conversation between Jared and the interviewer.

Interviewer: Can you guys talk about what you are trying to do before you continue?

Jared: $\quad U h, I$ took this $\rho L$ and converted to this right here which is $\rho(x)$. And then multiply by $d$, uh, small distance $x$, each of the little resistivity? It's all those little pieces going up together. And so it's the integral from 0 toL, of $e^{-x / L} d x$.

Interviewer: Can you explain more about this part, the integral part?

Jared: Basically this part right here (drew a rectangular box on the equation as shown in

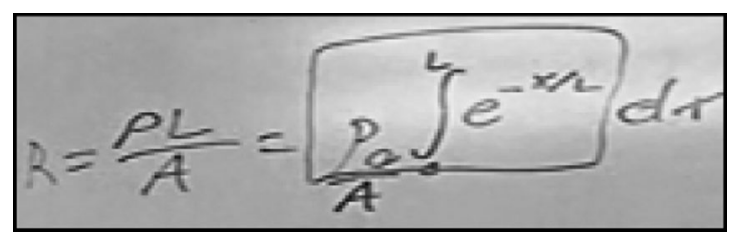

FIG. 13. Equation set up by Jared. 
Fig. 5) is the $\rho$, resistivity, and the $d x$ is the length, and uh, since it's not just multiplied by one small length, it's from 0 to $L$, so you get the whole length.

Interviewer: Uh huh.

Jared: $\quad$ And you are also adding up all of the resistivities together.

Jared explained that each small piece was represented by " $d x$ " and by using integration, "all those little pieces going up together." Jared seemed to view the use of integral in this physical situation as adding up small quantities. By saying "I took this $\rho L$ and converted to this right here," Jared mapped the basic resistance equation and the summation idea into the structure of the basic resistance equation. He interpreted the variable of integration $d x$ as "little pieces" and the integral part in the rectangular box (Fig. 13) as "adding up all of the little resistivities." The expression in the rectangular box is neither a complete nor correct expression without $d x$. By saying "adding up all of the resistivities together" he seemed to have understood that an integral represents a sum. However, he did not seem to realize that the resistivity represents the property of this material and the resistivity at different points could not be added up in this physical scenario.

The blending diagram (Fig. 14) represents how Jared constructed his solution. There are three mental spaces involved in this blending process: symbolic space, math notion space, and physics space. The symbolic space contains the expression of $\rho(x)$ and the basic resistance equation. The math notion space contains the idea of integral $\int$ as a sum and differential $d[]$ as a small quantity. The physics space contains physical quantities associated with the resistor, including the length, resistivity, and resistance.

When composing the blend, Jared mapped elements from input spaces to the blended space: the expression $\rho(x)$ and the mathematical notion of integral $\int$ as a sum was combined to construct an integral of $\rho(x)$, generating a new meaning of summing up little resistivities. Further, they seemed to map the idea of the differential $d[]$ as a

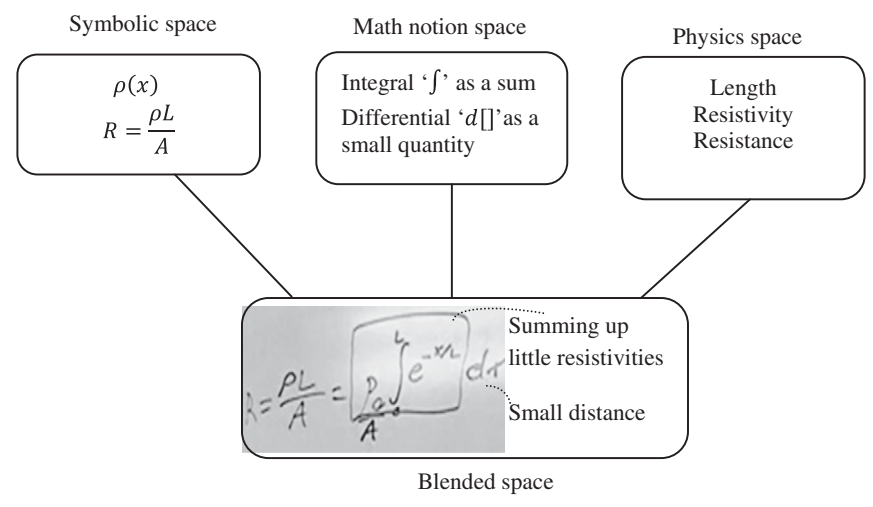

FIG. 14. Blend C. small amount of quantity from the mathematical notion space, and the length of cylinder from the physics space into the new space to construct the differential $d x$ as small distance or length. We speculate that the students mapped the structure of the basic resistance equation $(R=\rho L / A)$ into the blended space, in which the expression inside the box is considered as the total resistivity and $d x$ is considered as the length. In the blended space, Jared constructed an integral $R=\int_{0}^{L} \rho(x) d x / A$ which encapsulates an emergent meaning - adding up resistivities and distances. Jared tried to separate the structure of $\int_{0}^{L} \rho(x) d x$ into two partsresistivities and distances; however, the two parts are entangled in a way that, as you integrate, you "add up the resistivities" and "all the little pieces going up together." It is only in the blended space that this "splitting" and "entanglement" can coexist. Jared recruited the structure of the equation from the symbolic mental space in combination of the "integral as a summation" idea from the mathematics notion space to construct the solution. In other words, the organizing structure of the blend results primarily from an integration of structural elements from the symbolic space and mathematical notion space. Guided by the notion of integration as adding up small quantities in the mathematical notion space, Jared incorrectly mapped the resistivity instead of the resistance as the quantity being added up. When making this one-way mapping, we suspect that Jared was not aware of the structure or concepts in the physics space: resistivity is an intensive physical quantity that characterizes the variation of the material in different parts of the object in terms of its ability to resist the flow of current; thus, it is inappropriate to add up resistivity physically. Unlike the previous two blends where students tended to map elements from both symbolic space and physics space to the structure of mathematical notion space, Jared organized his solution based on the blending of the structure from both symbolic and mathematical notion space.

\section{Blend D}

In the following episode, two students, David and Alice, used an approach which is similar to the typical approach described in Fig. 4. They first chopped the cylindrical resistor into infinitely thin disks and then added the resistance due to each thin disk to get the total resistance. The following conversation occurred as soon as they started to solve this problem.

David: $\quad$ Okay, I think we need to separate this into

Alice: Yeah.

David: $\quad$ Little disks. Disks are fun.

Interviewer: So what made you guys think you need to separate it into disks?

David: $\quad$ In this case, because the resistivity changes as the length increases (see gesture in Fig. 16). 


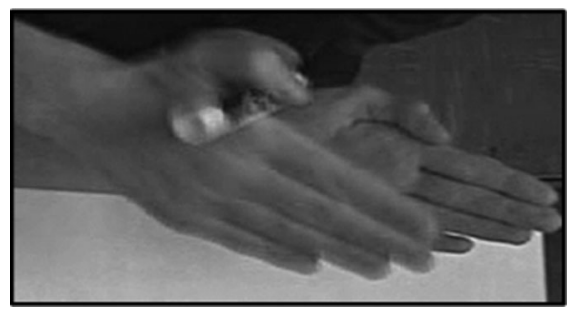

FIG. 15. David's gesture about "separating into pieces."

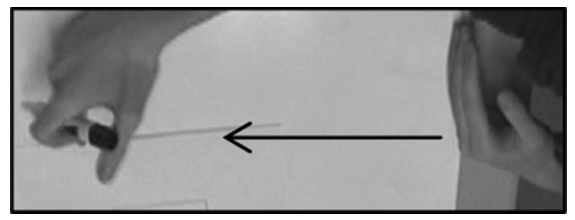

FIG. 16. David's gesture about "increasing resistivity."

Alice: $\quad$ So that's the sum, when we do that, it is just an integral.

David: $\quad$ It is different here than it is here (see gesture in Fig. 17). So, the easiest way is to picture it as infinitesimal thing at different values and then add up all the different values.

After this short conversation, they set up the differential form $d R$, which is the infinitesimal resistance due to a thin section of the cylinder of thickness $d x$. They then set up the expression for total resistance $R$ by taking the integral of $d R$. Their solution is shown in Fig. 18. Then the interviewer prompted them to explain their thinking. During his explanation, David also drew a picture as shown in Fig. 19.

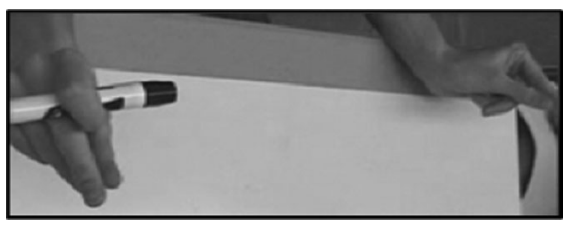

FIG. 17. David's gesture about "different values."

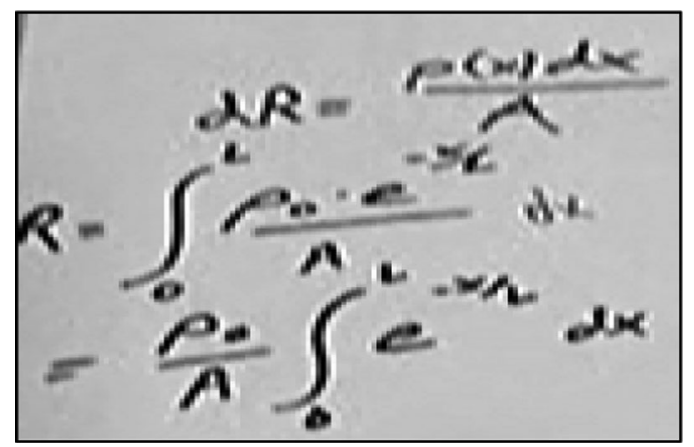

FIG. 18. Solution from David and Alice.

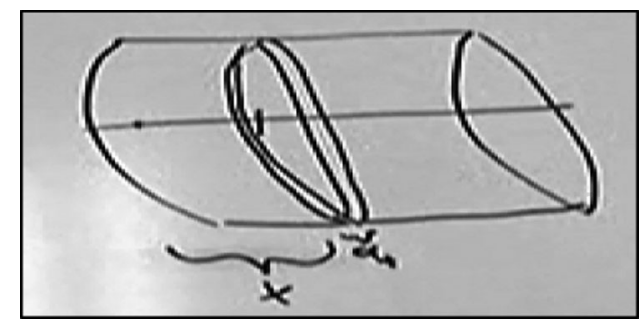

FIG. 19. Picture drawn by David.

Interviewer: Okay. Can you guys explain how you got this equation (referring to the integral equation in Fig. 15)?

Alice: Okay. Well, we are taking that one (the basic resistance equation) and so... but since we have to sum up a bunch of little pieces, you have to get little pieces, little piece of $R$, and then our function for $\rho$, as $\rho$ changes with respect to $x$, and where $x$ is little pieces of length. And so we cannot use whole of it, we have to use $d x$, and this is just the area (points to the equations in Fig. 18). So, it's our resistivity, (laughs) our resistance, is dependent on our length...

Interviewer: Okay. If you are going to explain your solution to your classmates, or friends, how would you explain it?

David: $\quad$ Well, I guess that would be ... Since we have a material that has a varying density, we can separate it into small cylinders, each one having a different density depending on their location $x$. So, starting out with the basic equation, we would find the resistance of each individual cylinder, which is $d R$, and that would be our little piece of resistance. And when you sum up those resistances from the left side to the right side, 0 to L, um, you would get the total resistance.

David started with "separate this into like, little pieces," and used his hands (Fig. 15) to imitate the action of "chopping the whole cylinder into little pieces." $\mathrm{He}$ explained that "resistivity changes as length changes" and indicated the resistivity was different at different positions by sweeping his hand across using the gestures shown in Figs. 16 and 17. The students realized that they needed to do a "sum" or "integral" to "add up all the different values." In the above episode, the students first analyzed the physical situation, then they developed a story about it using words and gestures, and finally they began to apply formal mathematics to set up an integral. When setting up the integral expression, they first found $d R$, which was the "little piece of $R$ " or "resistance of each individual 


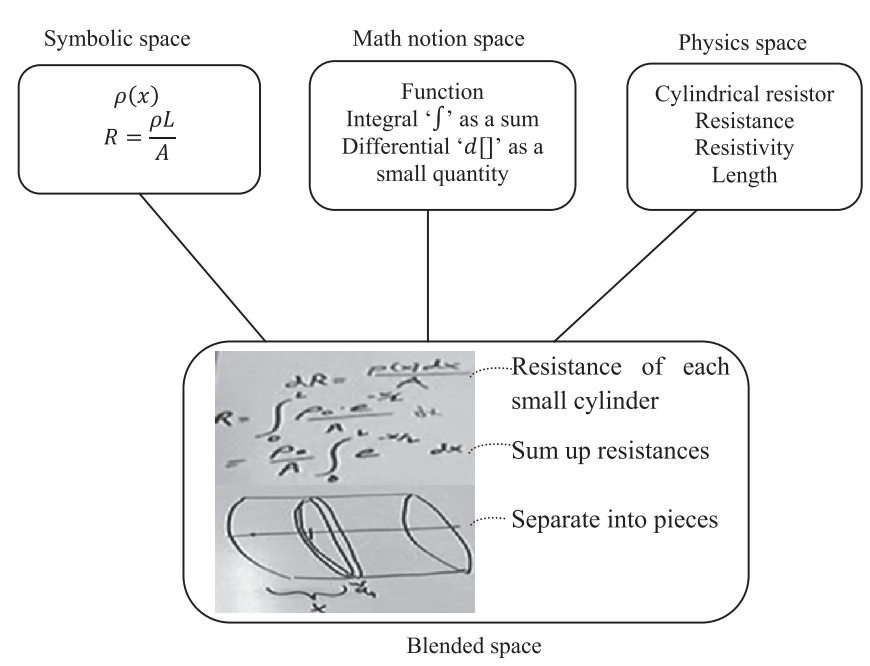

FIG. 20. Blend D.

cylinder," then they "summed up those resistances" using an integral to get the total resistance. He realized each resistor had a certain amount of resistance and could be added up. We construct a blending diagram (Fig. 20) to describe how students applied their mathematics knowledge in this physical situation to set up an integral.

The blending diagram (Fig. 20) includes three input mental spaces: symbolic space, math notion space, and physics space. In the upper left of the blending diagram, the symbolic space contains the expression of resistivity function and the basic resistance equation; the math notion space involves students' knowledge about function, integral as a sum, and differential as a small quantity. In the upper right, the physics space is the physical scenario containing a cylindrical resistor and the physical quantities of resistivity, resistance, and length. The three input spaces were integrated to create the blend. The expression of $\rho(x)$, the mathematical notion of function as a varying quantity depending on another quantity, and the cylinder were associated together in the blended space, generating a meaning of "resistivity changes as the length increases" or resistivity is different at different lengths. When David visualized the change of resistivity as the length changes in the physics space, it also cued him to "separate it into small cylinders" and apply the concept of integration. Then, the basic resistance equation was associated with the small cylinders, along with the mathematical notion of differential $d[$ ] as representing a small amount of quantity. A new structure emerged from this blend: chopping up total resistance $R$ and adding up small pieces of resistance $d R$. Unlike the other three blends which primarily involved a one-way mapping, this blend involved a two-way mapping among the three input spaces. The solution was organized based on the blend of the structural elements from all three input spaces.

\section{E. Summary of the four blends}

Our four blends are identified based on our observations of student work. As shown in Table I, those four blends involve three input mental spaces as well as different organizing structures. The organizing structure or frame describes the way in which students organize the knowledge elements in the blended space. We identified three input mental spaces involved in students' construction of solution. Blends A and B primarily involved a one-way mapping, in which the organizing structure was recruited from the mathematical notion space. In blend A, students had the idea of an integral as adding up quantities; however, they imported elements from both symbolic and math notion spaces, without mapping back to the physics space to justify whether the symbol or equation represented the appropriate quantity that they wanted to add up. The emergent structure from this blend is that the mathematical expression $\int_{0}^{L}[\rho(x) L / A] d x$ contains the meaning of the total resistance as the sum of a varying resistance across the length over which the resistivity varies. In blend B, the

TABLE I. Description of the four blends.

\begin{tabular}{|c|c|c|c|}
\hline Blend & Organizing frame & Emergent meaning or structure & Representations \\
\hline A & $\begin{array}{l}\text { Integral as a sum (from math notion } \\
\text { space) }\end{array}$ & $\begin{array}{l}\int_{0}^{L}[\rho(x) L / A] d x: \text { Total resistance is the sum } \\
\text { of a varying resistance across the length } \\
\text { over which the resistivity varies }\end{array}$ & Primarily algebraic \\
\hline B & $\begin{array}{l}\text { Differential as taking the derivative of } \\
\text { a function (from math notion space) }\end{array}$ & $\begin{array}{l}\int d R: \text { Total resistance is the integral of the } \\
\text { differential resistance-the derivative of a } \\
\text { resistance function }\end{array}$ & Primarily algebraic \\
\hline $\mathrm{C}$ & $\begin{array}{l}\text { Structure of basic resistance equation } \\
\text { and integral as a sum (a blend of } \\
\text { symbolic and math notion spaces) }\end{array}$ & $\begin{array}{l}\int_{0}^{L} \rho(x) d x / A: \int_{0}^{L} \rho(x) \text { represents adding up } \\
\quad \text { resistivities and } d x \text { as small distances being } \\
\quad \text { added up when integrating }\end{array}$ & Algebraic and narrative \\
\hline $\mathrm{D}$ & $\begin{array}{l}\text { Structure of basic resistance equation, } \\
\text { integral as a sum, chopping a resistor } \\
\text { (a blend of symbolic, math notion and } \\
\text { physics spaces) }\end{array}$ & $\begin{array}{l}\int d R \text { : Chopping up total resistance } R \text { and } \\
\text { adding up small pieces of resistance } d R\end{array}$ & $\begin{array}{l}\text { Algebraic, narrative, } \\
\text { pictorial, and heavy } \\
\text { use of gesturing }\end{array}$ \\
\hline
\end{tabular}


student mainly relied on his notion of differential as taking the derivative of a function and integration as the reverse of the differentiation action. His solution was based on mathematical manipulations without making connections to physical situations. This blend conveys the meaning that the mathematical expression $\int d R$ represents the total resistance, which is the integral of the differential resistance-the derivative of a resistance function. Blends A and B primarily involve algebraic representations, in which students focus on computation and manipulating mathematical symbols, and seldom connect these with the underlying physics.

When constructing blend C, Jared blended the structure of the equation from the symbolic mental space and the "integral as a summation" idea from the mathematics notion space. Then the elements in the physics space were mapped into the blended space and organized under the blended structure. Jared constructed the mathematical expression for the total resistance as $\int_{0}^{L} \rho(x) d x / A$, containing a meaning that $\int_{0}^{L} \rho(x)$ represents adding up resistivities and $d x$ as small distances being added up when integrating. Following this blend, the students set up an integral expression which was mathematically correct but was based on an incorrect physical principle; i.e., the resistivity at different parts of an object should be added up. In addition to algebraic representation, Jared also attempted to develop a story of mathematical symbols; i.e., $d x$ is small distance and small distances are added together when integrating. We describe this as the narrative representation. In blend D, students did not just recruit the structure from one input mental space; instead, they created a new structure based on the blending of structures from all three input spaces. A new meaning emerges from this blend-chopping up the object and adding up the resistance $d R$ of each of the small pieces to find the total resistance $R$. Students used multiple representations, including algebraic, narrative, pictorial presentations, as well as gesturing while creating this blend.

Overall, blend D is the most productive one among the four blends that the students created. This blend involves a much higher level of blending of students' mathematics and physics knowledge. In the blended space, students created a new structure of chopping and adding resistance. Blend $\mathrm{C}$ involves the inappropriate blending of knowledge in the symbolic space and mathematical notion space. Jared appeared to map his solution to the physics space when he explained $d x$ as small distances. He set up an integral equation which was mathematically equivalent to the correct answer, but the inherent physical structure was incorrect. In blend B, the organizing structure was based on the notion of differential as taking the derivative of a function. This blend is unproductive for setting up integrals since the student tended to find the function in an effortless way-simply substituting the resistivity function into the basic resistance equation. This blending is based primarily on a one-way mapping from the symbolic and physics spaces to the mathematical notion space. Similar to blend $\mathrm{B}$, blend $\mathrm{A}$ also involved a one-way mapping from the symbolic and physics spaces to the mathematical notion space. Students had the notion of an integral as adding up quantities; however, they built up a resistance function as the quantity being added up through mathematical substitution, which was an inappropriate way of bending knowledge from the symbolic and physics spaces. This too was also not a productive way for setting up integrals in the context of the physics problem at hand. The reason that we assert that some of these blends, such as blend $\mathrm{A}$, are unproductive is not because they failed to lead students to correctly set up the integral. Rather, we categorize these blends as unproductive blends because in constructing this blend students created mapping from the symbolic and physics spaces to the mathematical notion space without attending to the physical meaning of the situation.

\section{CONCLUSION AND IMPLICATIONS}

In this paper, we have illustrated how the conceptual blending framework can be used to analyze students' application of the integration concept in physics problem solving. From a large set of interview data, we selected data from five groups of students working on a problem situation and identified four different kinds of blends that emerged from the data as students constructed solutions to the problem.

In blends $\mathrm{A}$ and $\mathrm{B}$, students did not relate the mathematical concepts to the specific physical scenario of the problem. Rather, they mapped their mathematics knowledge to general physics concepts. Both blends involve an organizing frame which was primarily from the mathematical notion space. The organizing frame of blend A is the notion of an integral as adding up quantities and the organizing frame of blend B is the notion of a differential as taking the derivative of a function. The third type of blend-blend $\mathrm{C}$ - contains an organizing frame predominantly from the symbolic and math notion space. In the blended space, Jared constructed the notion of adding up the resistivities but did not seem to be making any connection to the physics concepts in order to distinguish between adding resistivities and adding resistances. Hence, the student eventually set up an expression which made sense to him, but the structure was not appropriate in the physics space. The last type of blend — blend D-contained an organizing frame based on the integration of knowledge elements from all three input spaces: the notion of integral as a sum in the math notion space and the cylindrical resistor in the physical space were associated with each other, resulting in chopping of the physical object and adding the quantity due to each small piece. Thus, the resistance equation in the symbolic space, the small piece of cylinder, and the notion of differential as a small 
quantity were blended to find the resistance of each small cylinder.

Bing and Redish [14] describe two distinct ways (i.e., single-scope blend and double-scope blend) in which students blended their mathematics and physics knowledge in the context of problems involving air drag and time travel. Neither of these problems involved mathematical integration. Physics problems that involve integration are mathematical dominated problems, and the kinds of blending are somewhat more complex. We illustrated four different kinds of blends that students created in one problem situation-finding the total resistance of a cylindrical resistor or varying resistivity. Transferring knowledge from calculus to physics is a challenging process for many introductory students, which is apparent from both our teaching experience as well as research on students' work. Analysis of students' work as they solve integration problems using this framework shows that the process of blending is complex and students blend their knowledge in many different ways. The difficulties that students experience seem to be not necessarily from a lack of prerequisite knowledge of mathematics, but rather from inappropriate blending of the knowledge of mathematics with their knowledge of physics concepts and the physical scenario at hand. Students appeared to have the required mathematical knowledge and were able to construct a solution in a physics context, but many of them were not able to blend their mathematics and physics knowledge in a productive way. We found that students who created three of the four observed blends had the notion of an integral as adding up quantities; however, they blended their knowledge in three mental spaces in different ways. One of these observed blends was a more powerful blend than the other two blends because it facilitated the combination of mathematical and physical ideas in a productive way. The description of students' work under the cognitive blending framework might help instructors better understand students' difficulties and the important features in student solutions.

During the interviews, we intended to have students solve physics problems in a somewhat natural environment in which the interviewer did not provide feedback on students' work or facilitate their problem solving process. At times, during the interviews, the interviewer (author $\mathrm{Hu}$ ) occasionally asked questions to probe students' thinking, but she was always mindful of trying not to change the ways in which students constructed their ideas. Under this environment, we found that the blends that students constructed involved very little integration of mathematics and physics knowledge. In other words, students relied heavily on their mathematical ideas but not their sense of the physical situations. Though the interviewer tried her best not to provide guidance on student work, we found that the interviewer did seem to affect students' construction of the blends. For example, a group of students discarded blend A, which they originally constructed, and shifted to blend $\mathrm{B}$ as the interviewer asked them to explain what $d R$ meant.

The results of this study also suggest that it is possible to steer students away from one blend to another when certain resources are provided. The construction of a blend is a dynamic process in which we observed that students changed their thinking and created a new blend when they were reflecting on their work or were asked questions that prompted them to activate different resources. For example, one student first constructed a blend similar to blend A and later switched to blend D as he saw a cylinder being sliced up drawn by one of the other students in his group.

Modeling student responses with the conceptual blending framework allows us to conduct a fine-grained analysis of student reasoning in our problem scenario. Here, we consider three mental spaces: symbol, math notion, and physics. This classification of mental spaces is based on characteristics of our problems as well as students' responses. We do not claim that this is the only way to classify students' mental spaces' however, it does help us to create a representation to describe student reasoning.

We analyzed students' application of calculus concepts in physics problems from the perspective of conceptual blending. The conceptual blending framework can be used to understand the creation of resources. In other words, the development of a resource may be described as a blend. A blend once constructed can become a resource for the student to be used in the future. For example, in blend $\mathrm{A}$, students constructed a mathematical expression $\int_{0}^{L}[\rho(x) L / A] d x$, which contains a meaning that the total resistance is the sum of a varying resistance across the length over which the resistivity varies. It can also be described as a mathematical resource (i.e., a symbolic form) in which the mathematical structure $\int_{[]}^{[]}[] d[]$ provides a way for students to associate mathematical symbols with their intuitive mathematical knowledge (i.e., conceptual schema). Jones [9] described the conceptual schema as "add up then multiply" - adding up one and then multiplying the resultant summation with the quantity represented by the differential. From the resources perspective, students applied the "add up then multiply" resource in this physics context. Instead of assuming that this resource preexists in students' minds and becomes activated in this context, we describe how this resource is constructed based on more generic resources such as the notion of an integral as adding up quantities. The power of the conceptual blending framework is that it provides a language for describing the process by which learners activate generic resources to create new resources to solve the problem at hand.

\section{ACKNOWLEDGMENTS}

This work is supported in part by the National Science Foundation Grant No. 0816207. 
[1] F. R. Yeatts and J. R. Hundhausen, Calculus and physics: Challenges at the interface, Am. J. Phys. 60, 716 (1992).

[2] E.F. Redish, in Proceedings of the Conference on World View on Physics Education in 2005: Focusing on Change, Delhi, India (to be published).

[3] L. Cui, N. S. Rebello, P. R. Fletcher, and A. G. Bennett, in Proceedings of the Annual Meeting of the National Association for Research in Science Teaching, San Francisco, 2006 (to be published).

[4] D. Nguyen and N. S. Rebello, Students' difficulties with integration in electricity, Phys. Rev. ST Phys. Educ. Res. 7, 010113 (2011).

[5] G. Fauconnier and M. Turner, Conceptual integration networks, Cogn. Sci. 22, 133 (1998).

[6] D. C. Meredith and K. A. Marrongelle, How students use mathematical resources in an electrostatics context, Am. J. Phys. 76, 570 (2008).

[7] B.R. Wilcox, M.D. Caballero, R.E. Pepper, and S. J. Pollock, Upper-division student understanding of Coulomb's law: Difficulties with continuous charge distributions , AIP Conf. Proc. 1513, 418 (2013).

[8] B. L. Sherin, How students understand physics equations, Cognit. Instr. 19, 479 (2001).

[9] S. R. Jones, Ph.D. thesis, University of Maryland, 2010.

[10] J. Tuminaro and E. Redish, Elements of a cognitive model of physics problem solving: Epistemic games, Phys. Rev. ST Phys. Educ. Res. 3, 020101 (2007).

[11] A. Collins and W. Ferguson, Epistemic forms and epistemic games: Structures and strategies to guide inquiry, Educ. Psychol. 28, 25 (1993).
[12] E. Gire, D.-H. Nguyen, and N. S. Rebello, in Proceedings of the 2011 National Association for Research in Science Teaching Annual Meeting, Orlando, 2011 (to be published).

[13] G. Fauconnier and M. Turner, The Way We Think: Conceptual Blending and the Mind's Hidden Complexities (Perseus Books Group, New York, 2002).

[14] T.J. Bing and E.F. Redish, The cognitive blending of mathematics and physics knowledge, AIP Conf. Proc. 883, 26 (2007).

[15] G. Fauconnier and M. Turner, in Conceptual Structure, Discourse, and Language, edited by A. Goldberg (Cambridge University Press, Cambridge, England, 1996), pp. 1-23.

[16] S. Coulson and T. Oakley, Blending basics, Cogn. Ling. 11, 175 (2001).

[17] M.C. Wittmann, in Proceedings of the 9th International Conference of the Learning Sciences, Chicago, 2010 (ICLS 2010), Vol. 1, pp. 659-666 [arXiv:1008.0216v1].

[18] M. Zandieh, J. Knapp, and K. H. Roh, in Proceedings of the 11th Conference of the SIGMAA and on RUME, San Diego, 2008 (to be published).

[19] J. Tuminaro, Ph.D. thesis, University of Maryland, 2004.

[20] P. V Engelhardt, E. G. Corpuz, D. J. Ozimek, and N. S. Rebello, The teaching experiment-What it is and what it isn't, AIP Conf. Proc. 720, 157 (2004).

[21] C. M. Sorensen, A. D. Churukian, S. Maleki, and D. A. Zollman, The new studio format for instruction of introductory physics, Am. J. Phys. 74, 1077 (2006).

[22] F. Marton, Phenomenography: A research approach to investigating different understandings of reality, J. Thought 21, 28 (1986). 